\title{
Variational Bayesian Approach in Model-Based Iterative Reconstruction for 3D X-Ray Computed Tomography with Gauss-Markov-Potts Prior ${ }^{\dagger}$
}

\author{
Camille Chapdelaine ${ }^{1, * \mathbb{D}}$, Ali Mohammad-Djafari ${ }^{2}$, Nicolas Gac ${ }^{2}$ and Estelle Parra ${ }^{1}$ \\ 1 SAFRAN SA, Safran Tech, Pôle Technologie du Signal et de l'Information, 78772 Magny-Les-Hameaux, \\ France; estelle.parra@safrangroup.com \\ 2 Laboratoire des signaux et systèmes, CNRS, CentraleSupélec-Université Paris-Saclay, 91190 Gif-sur-Yvette, \\ France; ali.mohammad-djafari@12s.centralesupelec.fr (A.M.-D.); nicolas.gac@12s.centralesupelec.fr (N.G.) \\ * Correspondence: camille.chapdelaine@safrangroup.com \\ + Presented at the 39th International Workshop on Bayesian Inference and Maximum Entropy Methods in \\ Science and Engineering, Garching, Germany, 30 June-5 July 2019.
}

Published: 21 November 2019

check for updates

\begin{abstract}
D X-ray Computed Tomography (CT) is used in medicine and non-destructive testing (NDT) for industry to visualize the interior of a volume and control its healthiness. Compared to analytical reconstruction methods, model-based iterative reconstruction (MBIR) methods obtain high-quality reconstructions while reducing the dose. Nevertheless, usual Maximum-A-Posteriori (MAP) estimation does not enable to quantify the uncertainties on the reconstruction, which can be useful for the control performed afterwards. Herein, we propose to estimate these uncertainties jointly with the reconstruction by computing Posterior Mean (PM) thanks to Variational Bayesian Approach (VBA). We present our reconstruction algorithm using a Gauss-Markov-Potts prior model on the volume to reconstruct. For PM calculation in VBA, the uncertainties on the reconstruction are given by the variances of the posterior distribution of the volume. To estimate these variances in our algorithm, we need to compute diagonal coefficients of the posterior covariance matrix. Since this matrix is not available in 3D X-ray CT, we propose an efficient solution to tackle this difficulty, based on the use of a matched pair of projector and backprojector. In our simulations using the Separable Footprint (SF) pair, we compare our PM estimation with MAP estimation. Perspectives for this work are applications to real data as improvement of our GPU implementation of SF pair.
\end{abstract}

Keywords: Computed Tomography, Gauss-Markov-Potts, variational Bayesian approach, Separable Footprint

\section{Introduction}

In 3D X-ray CT, MBIR methods enforce a prior model on the volume to image, so the reconstruction quality is enhanced compared to filtered backprojection (FBP) methods [1], and the dose can be reduced [2]. Smoothing and edge-preserving priors, such as total variation regularization [3,4], Gauss-Markov-Potts prior model [5] or sparsity-inducing priors in a wavelet or learnt transform domain [6-8], have provided promising results for the development of MBIR methods in medicine and NDT for industry. Due to the high dimension and to the fact that the reconstruction problem is ill-posed [9], exact estimation of the unknown volume is not available [10]. As a consequence, uncertainties on the estimation are a desirable tool for the analysis of the reconstructed volume.

After the reconstruction has been performed, an iterative method to estimate the uncertainties is proposed in [10]. Nevertheless, its high computational cost makes it only applicable to a few voxels of interest [10]. Since MBIR methods mostly estimate the maximum of the posterior distribution of 
the unknowns (MAP), confidence regions can be computed following the reconstruction [11] but this procedure is difficult to apply for discrete-continuous channels estimation, such as joint reconstruction and segmentation [5]. For this reason, in this paper, we propose to compute Posterior Mean (PM) rather than MAP. For PM estimator, the uncertainties on the reconstruction correspond to the variances. Our algorithm estimates these variances jointly with the reconstruction based on variational Bayesian approach (VBA) [12,13].

In the following, we first present our reconstruction algorithm based on VBA, applied with a Gauss-Markov-Potts prior model on the volume to reconstruct [5]. To implement this algorithm, the main difficulty is the computation of diagonal coefficients of the posterior covariance matrix, which are linked to projection and backprojection operators $(\mathrm{P} / \mathrm{BP})$ : we solve this problem thanks to the use of a matched pair which is here the Separable Footprint (SF) [14]. We present simulation results and compare the obtained reconstruction with the one given by joint maximization a posteriori (JMAP) $[5,15]$. To the best of our knowledge, this work is the first attempt to apply VBA to a very general 3D inverse problem such as 3D X-ray CT.

\section{Variational Bayesian Approach}

We consider a cone-beam acquisition process : X-rays are sent from a source through the object to control and hit a flat detector which measures the decrease of intensity they have undergone inside the volume. Several perspectives of the volume are acquired by rotating the object around its vertical axis. The $M$ collected measurements $\boldsymbol{g}$ are called the projections and are connected to volume $\boldsymbol{f}$, of size $N$, by the linear forward model taking uncertainties into account [16]

$$
g=H \boldsymbol{f}+\zeta
$$

where $\boldsymbol{H}$ is called the projection operator. Its adjoint $\boldsymbol{H}^{T}$ is the backprojection operator [14]. Since both the data and the volume are huge, matrix $\boldsymbol{H}$, which is size $M \times N$, is not storable in memory. Consequently, successive projections and backprojections in MBIR methods are computed on-the-fly [14,15]. Uncertainties $\zeta$ are zero-mean Gaussian [16]

$$
p\left(\zeta_{i} \mid \rho_{\zeta_{i}}\right)=\mathcal{N}\left(\zeta_{i} \mid 0, \rho_{\zeta_{i}}^{-1}\right), \forall i \in\{1, \ldots, M\}
$$

Precisions $\boldsymbol{\rho}_{\zeta}=\left(\rho_{\zeta_{i}}\right)_{i}$ are assigned Gamma conjugate prior [5] :

$$
p\left(\rho_{\zeta_{i}} \mid \alpha_{\zeta_{0}}, \beta_{\zeta_{0}}\right)=\mathcal{G}\left(\rho_{\zeta_{i}} \mid \alpha_{\zeta_{0}}, \beta_{\zeta_{0}}\right), \forall i
$$

The prior model on the volume is a Gauss-Markov-Potts prior which consists in labelling each voxel $j$ according to its material $z_{j}=k \in\{1, \ldots, K\}$, where $K$ is the number of materials. Then, the distribution of value $f_{j}$ of voxel $j$ depends on its material $z_{j}$ :

$$
f_{j} \sim \mathcal{N}\left(m_{k}, \rho_{k}^{-1}\right) \text { if } z_{j}=k, \forall j \in\{1, \ldots, N\} .
$$

Means $\boldsymbol{m}=\left(m_{k}\right)_{k}$ and inverses $\boldsymbol{\rho}=\left(\rho_{k}\right)_{k}$ of variances of the classes have to be estimated and are assigned conjugate priors [5] :

$$
\left\{\begin{array}{l}
p\left(m_{k} \mid m_{0}, v_{0}\right)=\mathcal{N}\left(m_{k} \mid m_{0}, v_{0}\right) \\
p\left(\rho_{k} \mid \alpha_{0}, \beta_{0}\right)=\mathcal{G}\left(\rho_{k} \mid \alpha_{0}, \beta_{0}\right)
\end{array}, \forall k\right.
$$


A Potts model is assigned to labels $z$ in order to favour compact regions in the volume [5] : denoting by $\mathcal{V}(j)$ the neighbourhood of voxel $j$, we have, according to Hammersley-Clifford theorem [17],

$$
p\left(\boldsymbol{z} \mid \boldsymbol{\alpha}, \gamma_{0}\right) \propto \exp \left[\sum_{j=1}^{N}\left(\sum_{k=1}^{K} \alpha_{k} \delta\left(z_{j}-k\right)+\gamma_{0} \sum_{i \in \mathcal{V}(j)} \delta\left(z_{j}-z_{i}\right)\right)\right] .
$$

From our prior model $\mathcal{M}$, the posterior distribution of unknowns $\psi=\left(\boldsymbol{f}, \boldsymbol{z}, \boldsymbol{\rho}_{\zeta}, \boldsymbol{m}, \boldsymbol{\rho}\right)$ is given by Bayes' rule [5]

$$
p\left(\boldsymbol{f}, \boldsymbol{z}, \boldsymbol{\rho}_{\zeta}, \boldsymbol{m}, \boldsymbol{\rho} \mid \boldsymbol{g} ; \mathcal{M}\right) \propto p\left(\boldsymbol{g} \mid \boldsymbol{f}, \boldsymbol{\rho}_{\zeta}\right) p(\boldsymbol{f} \mid \boldsymbol{z}, \boldsymbol{m}, \boldsymbol{\rho}) p\left(\boldsymbol{z} \mid \boldsymbol{\alpha}, \gamma_{0}\right) p\left(\boldsymbol{\rho}_{\zeta} \mid \alpha_{\zeta_{0}}, \beta_{\zeta_{0}}\right) p\left(\boldsymbol{m} \mid m_{0}, v_{0}\right) p\left(\boldsymbol{\rho} \mid \alpha_{0}, \beta_{0}\right),
$$

where $\alpha=\left(\alpha_{k}\right)_{k}$. Based on this distribution, JMAP can be performed [5] but does not provide uncertainties on the result. MCMC methods for joint computation of the means and the variances of the posterior distribution are too computationally costly for 3D applications $[5,18]$. For this reason, we apply VBA which consists in approximating the true posterior distribution $p$ by a simpler distribution $q$ on which posterior means and variances can be easily estimated. Approximating distribution $q$ minimizes Kullback-Leibler (KL) divergence $K L(q \| p)$ on a chosen set of simple distributions [12]. The choice we make for $q$ is a factorizable approximation, which only preserves a dependence between value $f_{j}$ of voxel $j$ and its label [19]:

$$
q\left(\boldsymbol{f}, \boldsymbol{z}, \boldsymbol{\rho}_{\zeta^{\prime}}, \boldsymbol{m}, \boldsymbol{\rho}\right)=\prod_{j=1}^{N} q_{f_{j}}\left(f_{j} \mid z_{j}\right) \times \prod_{j=1}^{N} q_{z_{j}}\left(z_{j}\right) \times \prod_{i=1}^{M} q_{\rho_{\zeta_{i}}}\left(\rho_{\zeta_{i}}\right) \times \prod_{k=1}^{K} q_{m_{k}}\left(m_{k}\right) \times \prod_{k=1}^{K} q_{\rho_{k}}\left(\rho_{k}\right) .
$$

Minimizing KL divergence with respect to each factor while fixing the others leads to $[13,19]$

$$
\left\{\begin{array}{l}
q_{f_{j}}\left(f_{j} \mid z_{j}=k\right)=\mathcal{N}\left(f_{j} \mid \tilde{m}_{j k}, \tilde{v}_{j k}\right) \\
q_{z_{j}}(k) \propto \exp \left[\tilde{\alpha}_{j k}+\gamma_{0} \sum_{i \in \mathcal{V}(j)} q_{z_{i}}(k)\right], \forall k \\
q_{\rho_{\zeta_{i}}}\left(\rho_{\zeta_{i}}\right)=\mathcal{G}\left(\rho_{\zeta_{i}} \mid \tilde{\alpha}_{\zeta_{0_{i}}}, \tilde{\beta}_{\tau_{0_{i}}}\right) \\
q_{m_{k}}\left(m_{k}\right)=\mathcal{N}\left(m_{k} \mid \tilde{m}_{0_{k}}, \tilde{v}_{0_{k}}\right) \\
q_{\rho_{k}}\left(\rho_{k}\right)=\mathcal{G}\left(\rho_{k} \mid \tilde{\alpha}_{0_{k}}, \tilde{\beta}_{0_{k}}\right)
\end{array}\right.
$$

The VBA algorithm turns into the iterative updating of the parameters of these distributions with respect to the others. The updating formulae and the order of their applications are given in [13]. In particular, at iteration $t$, the variances of the approximating distribution for the volume are updated by

$$
\tilde{v}_{j k}^{(t)}=\left(\frac{\tilde{\alpha}_{0_{k}}^{(t-1)}}{\tilde{\beta}_{0_{k}}^{(t-1)}}+\left[\boldsymbol{H}^{T} \tilde{\boldsymbol{V}}_{\zeta}^{-1} \boldsymbol{H}\right]_{j j}\right)^{-1}
$$

where $\tilde{\boldsymbol{V}}_{\zeta}=\operatorname{diag}\left[\tilde{\boldsymbol{v}}_{\zeta}\right]$ and $\tilde{v}_{\zeta i}=\frac{\tilde{\beta}_{\zeta_{0}}^{(t-1)}}{\tilde{\alpha}_{\zeta_{0_{i}}}^{(t-1)}}, \forall i$ [13]. Moreover, the updating formula for intensity parameter of the approximating Gamma distribution for $\rho_{\zeta_{i}}$ is [13]

$$
\tilde{\beta}_{\zeta_{0_{i}}}^{(t)}=\beta_{\zeta_{0}}+\frac{1}{2}\left(\left(g_{i}-[\boldsymbol{H} \tilde{\boldsymbol{m}}]_{i}\right)^{2}+\left[\boldsymbol{H} \tilde{\boldsymbol{V}} \boldsymbol{H}^{T}\right]_{i i}\right)
$$

where $\tilde{\boldsymbol{V}}=\operatorname{diag}[\boldsymbol{v}]$ and

$$
\left\{\begin{array}{l}
\tilde{m}_{j}=\sum_{k=1}^{K} \tilde{m}_{j k}^{(t)} q_{z_{j}}^{(t)}(k) \\
\tilde{v}_{j}=\sum_{k=1}^{K}\left[\tilde{v}_{j k}^{(t)}+\left(\tilde{m}_{j k}^{(t)}-\tilde{m}_{j}\right)^{2}\right] q_{z_{j}}^{(t)}(k)
\end{array}\right.
$$


To compute approximate posterior variances, formula (10) needs the computation of diagonal coefficients of $\boldsymbol{H}^{T} \tilde{\boldsymbol{V}}_{\zeta}^{-1} \boldsymbol{H}$, while formula (11) needs diagonal coefficients of $\boldsymbol{H} \tilde{\boldsymbol{V}} \boldsymbol{H}^{T}$. Both of these matrices imply projector and backprojector which are not in memory, contrary to 2D applications [19]. Therefore, in order to implement VBA for 3D X-ray CT, we need to find a way to compute diagonal coefficients in formulae (10) and (11) efficiently. We propose a strategy which is detailed in the next section.

\section{Computation of diagonal coefficients}

At one iteration of the algorithm, for any voxel $j$, diagonal coefficient used to compute $v_{j k}$ by (10) is

$$
d_{v_{j}}=\left[\boldsymbol{H}^{T} \tilde{\boldsymbol{V}}_{\zeta}^{-1} \boldsymbol{H}\right]_{j j}=\left\|\boldsymbol{H} \boldsymbol{e}^{(j)}\right\|_{\tilde{\boldsymbol{V}}_{\zeta}}^{2}
$$

where $e_{i}^{(j)}=\delta(j-i), \forall i$. As $\boldsymbol{d}_{v}=\left(d_{v_{j}}\right)_{j}$ has the size of a volume, formula (13) implies to compute $N$ projections, which is very long, even if the projector implemented on GPU is very fast. We calculated that, if we have to reconstruct a volume of size $N=256^{3}$ voxels from 64 projections of size $256^{2}$ pixels, and if one projection takes only 10 milliseconds, computing all dialgonal coefficients $d_{v_{j}}, \forall j$, for only one iteration of proposed VBA algorithm [13], would require more than 40 hours. Due to this huge computational cost, we prefer to consider the algebraic formula :

$$
d_{v_{j}}=\left[\boldsymbol{H}^{T} \tilde{\boldsymbol{V}}_{\zeta}^{-1} \boldsymbol{H}\right]_{j j}=\sum_{i=1}^{M} H_{i j}^{2} \tilde{v}_{\zeta_{i}}^{-1}, \forall j
$$

From this formula, diagonal coefficients $\boldsymbol{d}_{v}$ appear to be similar to a backprojection of $\tilde{\boldsymbol{v}}_{\zeta}^{-1}=\left(\tilde{v}_{\zeta_{i}}^{-1}\right)_{i}$, except that coefficients $H_{i j}$ are replaced by their squares $H_{i j}^{2}, \forall i$, j. Similarly, diagonal coefficients

$$
d_{\zeta_{i}}=\left[\boldsymbol{H} \tilde{\boldsymbol{V}} \boldsymbol{H}^{T}\right]_{i i}=\sum_{j=1}^{N} H_{i j}^{2} \tilde{v}_{j}, \forall i
$$

appear like a projection of volume $\tilde{\boldsymbol{v}}$, with $H_{i j}^{2}$ instead of $H_{i j}$. Given formulae (14) and (15), we implement a squared-projector $\boldsymbol{H}^{(2)}$ such that $H_{i j}^{(2)}=H_{i j}^{2}, \forall i, j$, and a squared-backprojector $\left(\boldsymbol{H}^{(2)}\right)^{T}$. Both are implemented exactly like the projector and the backprojector respectively. In order to ensure the validity of formulae (14) and (15), and therefore the convergence of our algorithm, we use a matched P/BP pair, which is here the Separable Footprint (SF) pair [14]. This pair is implemented on GPU as described in [15]. The same implementation is used for $\boldsymbol{H}^{(2)}$ and $\left(\boldsymbol{H}^{(2)}\right)^{T}$.

Thanks to these new operators, in one iteration of our algorithm, diagonal coefficients $d_{v_{j}}, \forall j$, are simultaneously computed by applying $\left(\boldsymbol{H}^{(2)}\right)^{T}$, which is very fast because it takes exactly the same time as a backprojection, instead of $N$ projections. Similarly, diagonal coefficients $d_{\zeta_{i}}, \forall i$, are simultaneously computed by applying $\boldsymbol{H}^{(2)}$, as fast as one projection, instead of $M$ backprojections.

Figure 1 shows diagonal coefficients of $\boldsymbol{H} \boldsymbol{H}^{T}$ and $\boldsymbol{H}^{T} \boldsymbol{H}$, computed by $\boldsymbol{H}^{(2)}$ and $\left(\boldsymbol{H}^{(2)}\right)^{T}$ respectively. Diagonal coefficients of $\boldsymbol{H} \boldsymbol{H}^{T}$ have the size of projections and are shown as it in Figure 1, while those of $\boldsymbol{H}^{T} \boldsymbol{H}$ are shown as a volume. We now apply our VBA algorithm to simulated data, and compare the estimated PM with JMAP. JMAP algorithm is described in [5] and applied with SF pair as we did in [15]. 


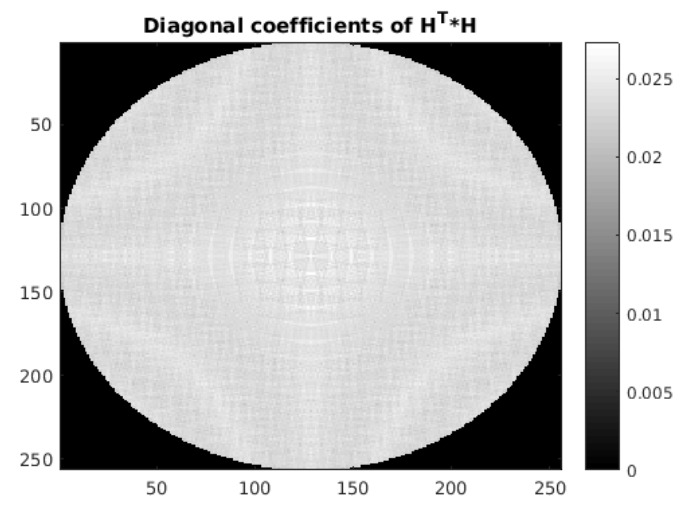

(a)

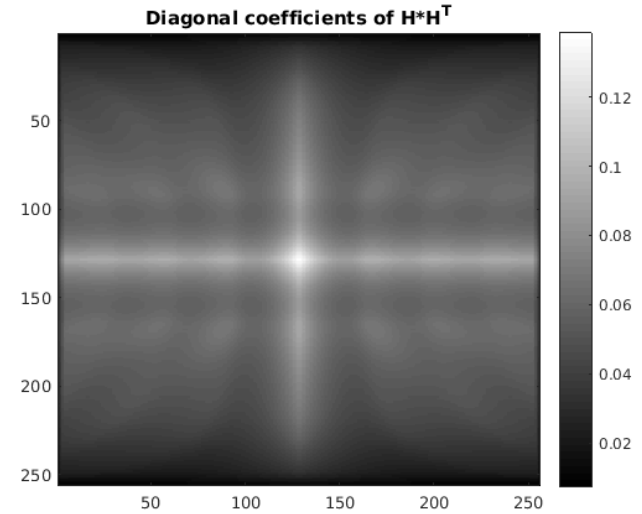

(b)

Figure 1. Diagonal coefficients of $\boldsymbol{H}^{T} \boldsymbol{H}(\mathbf{a})$ and $\boldsymbol{H} \boldsymbol{H}^{T}$ (b).

\section{Results}

The simulated phantom is of size $256^{3}$ voxels and contains $K=5$ classes. It is shown in Figure 2. We reconstruct this volume from 64 projections of size $256^{2}$ pixels, uniformly distributed over $[0,2 \pi]$. These projections are noisy with SNR equal to $20 \mathrm{db}$.

Parameters $\left(\alpha_{\zeta_{0}}, \beta_{\zeta_{0}}, \alpha_{0}, \beta_{0}\right)$ are fixed near Jeffreys' prior as in [13,19]. The strategies to fix other parameters $\alpha, \gamma_{0}, m_{0}$ and $v_{0}$ are explained in [13]. The values of the parameters for VBA are given in Table 1, excepted $m_{0}$ and $\alpha$ which are fixed automatically as in [5]. For our comparison, the parameters are the same for JMAP.

Table 1. Parameters for JMAP and VBA algorithms.

\begin{tabular}{llllllll}
\hline Parameters & $K$ & $\gamma_{0}$ & $v_{0}$ & $\alpha_{\zeta_{0}}$ & $\beta_{\zeta_{0}}$ & $\alpha_{0}$ & $\beta_{0}$ \\
\hline Values & 5 & 6 & 1 & $10^{-4}$ & $10^{-2}$ & $10^{-6}$ & $10^{-2}$ \\
\hline
\end{tabular}

The initialization of approximating distributions for VBA is described in [13]. This initialization requires initial volume and segmentation, obtained as explained in [13]. The same initialization is used for JMAP.

Figures 3 and 4 show the reconstructions obtained by JMAP and VBA respectively. They are compared with total-variation (TV) regularization. For TV, the reconstruction, shown in Figure 5, is obtained thanks to Primal-Dual Frank-Wolfe algorithm (PDFW) [20]. Thanks to the use of GaussMarkov-Potts prior model, JMAP and VBA reconstructions have compact and well-distinguishable regions, while contours are slightly blurred for TV. VBA reconstruction has smoother contours than JMAP.

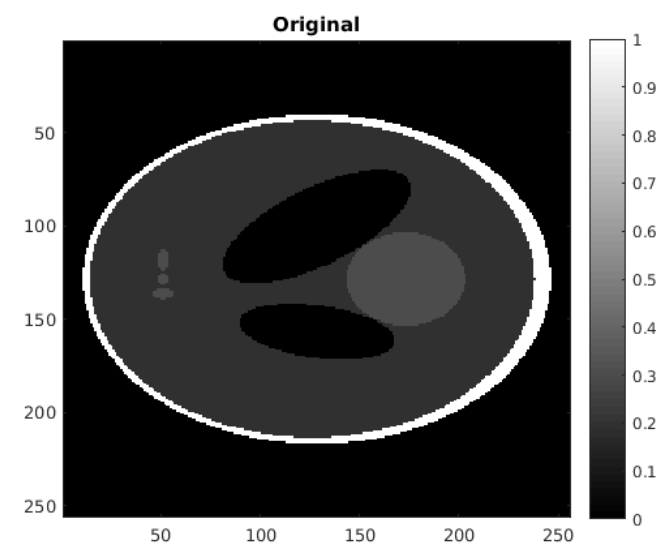

Figure 2. Original phantom. 


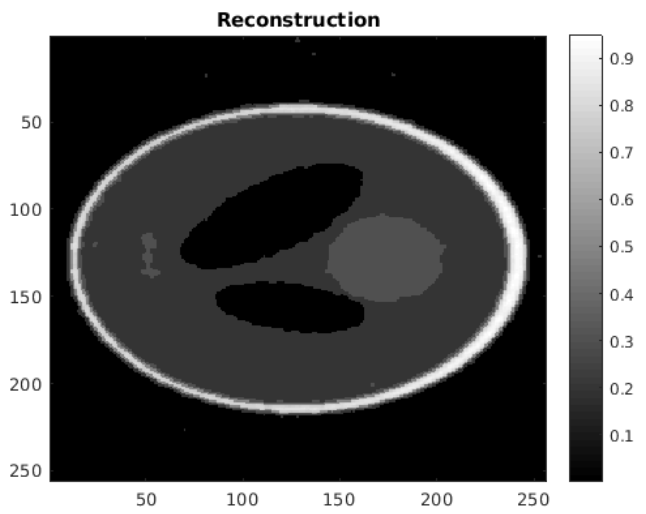

Figure 3. Reconstruction by JMAP.

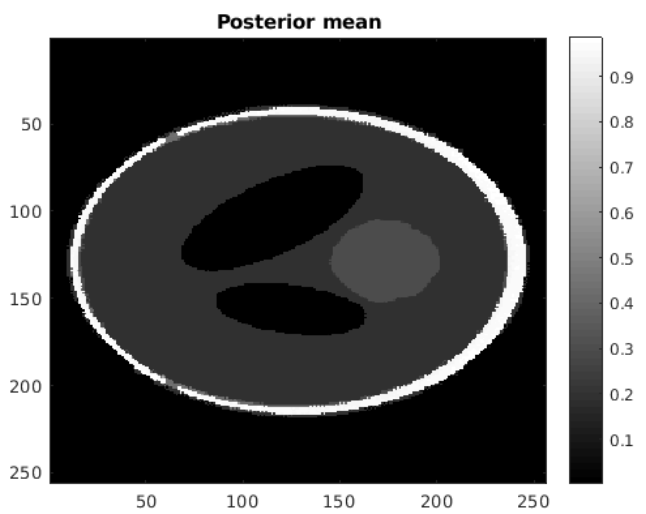

Figure 4. Reconstruction by VBA.

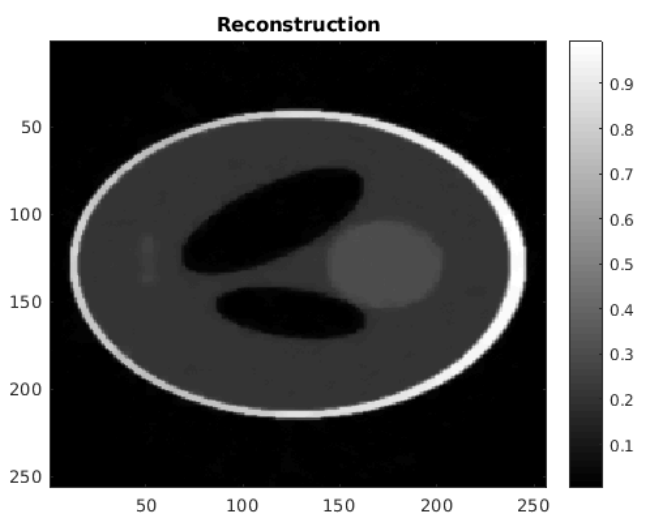

Figure 5. Reconstruction by PDFW.

For each reconstruction, the $\mathcal{L}_{2}$-relative error with respect to the original phantom is shown in Table 2. As we see in Figure 4, details are lost by VBA because of the factorized approximating distribution. Consequently, VBA has the highest error, while it is roughly the same for PDFW and JMAP. The variances of the posterior distribution of the volume estimated by VBA are shown in Figure 6. Unsurprisingly, the highest variances are on the thinest part of the phantom which is the bone. Nevertheless, the loss of details in the reconstruction is not highlighted by posterior variances. Indeed, uncertainties are known to be under-estimated in VBA when considering divergence $K L(q \| p)$ [12]. The stop criterion for PDFW is given in [20] and is minimized, while those for JMAP and VBA are maximized and given in $[5,13]$ respectively. For each algorithm, the evolution of stop criterion is shown in Figures 7-9 respectively. One iteration of JMAP contains 20 sub-iterations and few sub-iterations 
for segmentation step [5], while VBA and PDFW do not have sub-iterations [13,20]. Consequently, in Table 2, the computation time of VBA is much less than the one of JMAP and quite similar to the one of PDFW. Furthermore, during our experiments, we have noticed that, compared to JMAP, VBA has a higher sensitivity to the choice of the parameters, as to the number of iterations. Indeed, for a too large number of iterations of VBA, the reconstruction is over-regularized. This is a drawback of VBA compared to JMAP.

Moreover, the memory cost of VBA is much higher than the one of JMAP and PDFW. This makes VBA only applicable to small regions-of-interest (ROI), typically of size $256^{3}$. Based on a reconstruction of high quality (for instance, obtained by JMAP [5]), the reconstruction of ROI can be performed following the method of [21], as done for other MBIR methods [14]. This point will be covered in future works.

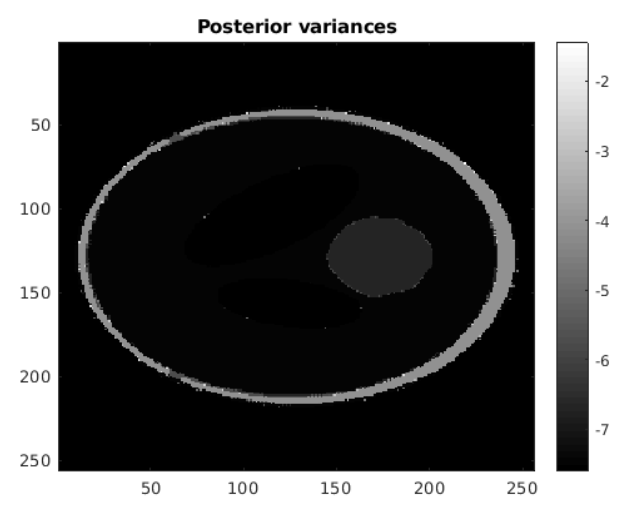

Figure 6. Variances (log) obtained by VBA

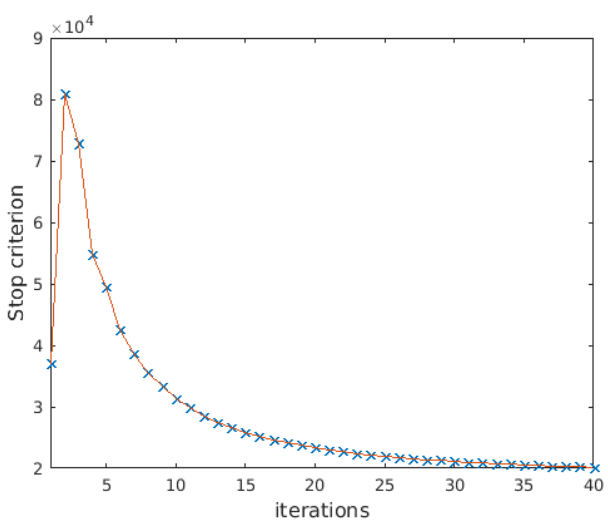

Figure 7. Convergence of PDFW

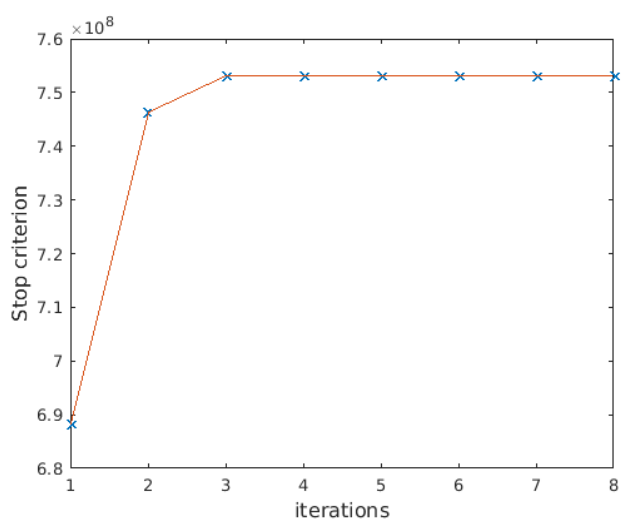

Figure 8. Convergence of JMAP 


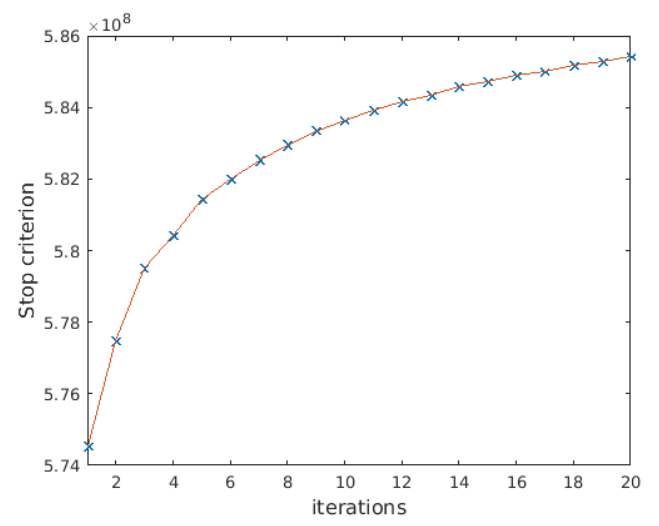

Figure 9. Convergence of VBA

Table 2. Comparaison of PDFW, JMAP and VBA algorithms.

\begin{tabular}{lll}
\hline Algorithm & $\mathcal{L}_{\mathbf{2}}$-Relative Error & Computation Time \\
\hline PDFW & $\mathbf{6 . 0} \%$ & $\mathbf{1 2 6 . 3 ~ s}$ \\
\hline JMAP & $9.1 \%$ & $751.6 \mathrm{~s}$ \\
\hline VBA & $13.5 \%$ & $150.0 \mathrm{~s}$ \\
\hline
\end{tabular}

\section{Conclusions and Perspectives}

In this paper, we have presented an application for 3D X-ray CT of variational Bayesian approach (VBA) with Gauss-Markov-Potts prior model. By computing posterior mean (PM) thanks to VBA, we have been able to jointly perform the reconstruction and the estimation of the posterior variances, which give the uncertainties on the reconstruction. To compute these variances, we have seen that the huge dimension in 3D X-ray CT hinders to easily get diagonal coefficients, due to the fact that projection and backprojection operators cannot be stored in memory. To tackle this problem, we have taken benefit from the use of a matched pair of projector and backprojector, which was the Separable Footprint (SF) one : based on this pair, we have implemented "squared" projector and backprojector which have enabled us to compute diagonal coefficients on-the-fly. The GPU implementation for these squared operators was the same we used for SF projector and backprojector.

Our tests on simulated data and comparison with joint maximization a posteriori (JMAP) have shown that VBA obtains smoother contours than JMAP and converges faster. Although the memory cost of VBA is higher than the one of JMAP, we have underlined that the algorithm can be applied to estimate the uncertainties in a region-of-interest (ROI). Future works will focus on applications to real and bigger data, as on optimization of GPU implementation of SF pair [15]. Other variational Bayesian algorithms will also be worth to study in order to improve the estimation of uncertainties.

Funding: This research was funded by CIFRE Grant 2016/0188 from French Agence Nationale de la Recherche et de la Technologie (ANRT).

\section{References}

1. Feldkamp, L.; Davis, L.; Kress, J. Practical cone-beam algorithm. JOSA A 1984, 1, 612-619.

2. Fessler, J.A. Statistical image reconstruction methods for transmission tomography. Hand. Med. Imaging 2000, 2, 1-70.

3. Sidky, E.Y.; Jakob, H.; Pan, X. Convex optimization problem prototyping for image reconstruction in computed tomography with the Chambolle \& Pock algorithm. Phys. Med. Biol. 2012, 57, 3065.

4. McGaffin, M.G.; Fessler, J.A. Alternating dual updates algorithm for X-ray CT reconstruction on the GPU. IEEE Trans. Comput. Imaging 2015, 1, 186-199. 
5. Chapdelaine, C.; Mohammad-Djafari, A.; Gac, N.; Parra, E. A 3D Bayesian Computed Tomography Reconstruction Algorithm with Gauss-Markov-Potts Prior Model and its Application to Real Data. Fundam. Inform. 2017, 155, 373-405.

6. Xu, Q.; Yu, H.; Mou, X.; Zhang, L.; Hsieh, J.; Wang, G. Low-dose X-ray CT reconstruction via dictionary learning. IEEE Trans. Med. Imaging 2012, 31, 1682-1697.

7. Vandeghinste, B.; Goossens, B.; Van Holen, R.; Vanhove, C.; Pižurica, A.; Vandenberghe, S.; Staelens, S. Iterative CT reconstruction using shearlet-based regularization. IEEE Trans. Nucl. Sci. 2013, 60, 3305-3317.

8. Zheng, X.; Ravishankar, S.; Long, Y.; Fessler, J.A. PWLS-ULTRA: An efficient clustering and learning-based approach for low-dose 3D CT image reconstruction. IEEE Trans. Med. Imaging 2018, 37, 1498-1510.

9. Idier, J. Bayesian Approach to Inverse Problems; John Wiley \& Sons: Hoboken, NJ, USA, 2008.

10. Fessler, J.A. Mean and Variance of Implicitly Defined Biased Estimators (such as Penalized Maximum Likelihood): Applications to Tomography. IEEE Trans. Image Process. 1996, 5, 493-506.

11. Pereyra, M. Maximum-A-Posteriori estimation with Bayesian Confidence Regions. SIAM J. Imaging Sci. 2017, 10, 285-302.

12. Pereyra, M.; Schniter, P.; Chouzenoux, E.; Pesquet, J.C.; Tourneret, J.Y.; Hero, A.O.; McLaughlin, S. A survey of stochastic simulation and optimization methods in signal processing. IEEE J. Select. Top. Signal Process. 2016, 10, 224-241.

13. Chapdelaine, C. Variational Bayesian Approach and Gauss-Markov-Potts prior model. arXiv 2018, arXiv:1808.09552.

14. Long, Y.; Fessler, J.A.; Balter, J.M. 3D forward and back-projection for X-ray CT using separable footprints. IEEE Trans. Med. Imaging 2010, 29, 1839-1850.

15. Chapdelaine, C.; Gac, N.; Mohammad-Djafari, A.; Parra, E. New GPU implementation of Separable Footprint Projector and Backprojector: First results. In Proceedings of the 5th International Conference on Image Formation in X-Ray Computed Tomography, 20-23 May 2018.

16. Sauer, K.; Bouman, C. A local update strategy for iterative reconstruction from projections. IEEE Trans. Signal Process. 1993, 41, 534-548.

17. Besag, J. Spatial interaction and the statistical analysis of lattice systems. J. R. Stat. Soc. Ser. B (Methodological) 1974, 36, 192-236.

18. Zhao, N.; Basarab, A.; Kouame, D.; Tourneret, J.Y. Joint segmentation and deconvolution of ultrasound images using a hierarchical Bayesian model based on generalized Gaussian priors. IEEE Trans. Image Process. 2016, 25, 3736-3750.

19. Ayasso, H.; Mohammad-Djafari, A. Joint NDT image restoration and segmentation using Gauss-MarkovPotts prior models and variational bayesian computation. IEEE Trans. Image Process. 2010, 19, 2265-2277.

20. Ongie, G.; Murthy, N.; Balzano, L.; Fessler, J.A. A Memory-Efficient Algorithm for Large-Scale Sparsity Regularized Image Reconstruction. In Proceedings of the Fifth International Conference on Image Formation in X-Ray Computed Tomography, 2018.

21. Ziegler, A.; Nielsen, T.; Grass, M. Iterative reconstruction of a region of interest for transmission tomography. Med. Phys. 2008, 35, 1317-1327.

(C) 2019 by the authors. Licensee MDPI, Basel, Switzerland. This article is an open access article distributed under the terms and conditions of the Creative Commons Attribution (CC BY) license (http:/ / creativecommons.org/licenses/by/4.0/). 